\title{
Pericarditis complicating a case of meningococcal meningitis
}

\author{
CLEANTH JONES \\ From Leicester General Hospital, Gwendolen Road, Leicester
}

The case is described of an 18-year-old schoolboy who developed pericarditis during the course of meningococcal meningitis; he had evidence of a preceding chronic meningococcaemia. Good recovery followed on systemic antibiotics only. The pericarditis was probably a direct septic complication of meningococcal meningitis.

Pericarditis is a rare complication of meningococcal meningitis, and extremely rare as the presenting feature. The case reported below describes pericarditis occurring during the course of meningococcal meningitis, in addition there was evidence of a preceding chronic meningococcaemia.

\section{Case report}

The patient was a previously fit, intelligent 18-yearold schoolboy. Three weeks before admission generalised malaise occurred associated with a moderate, dull, constant headache. These symptoms persisted, and 5 days before admission, he developed painful joints and a rash on the dorsum of the feet. During the night before admission he suddenly deteriorated, with increased headache, drowsiness, and severe prostration.

On admission the patient was drowsy, prostrate but apyrexial at $36 \cdot 5^{\circ} \mathrm{C}$. Blood pressure was $120 / 80$ $\mathrm{mmHg}(16.0 / 10.6 \mathrm{kPa})$ and pulse $100 /$ minute regular, with good volume. There was pronounced neck stiffness, positive Kernig's sign, and photophobia. He had generalised muscular weakness and a right ptosis. The metacarpophalangeal joints of the left index finger and right middle finger were reddened, swollen, and painful, with severe limitation of movement. There was a light brown nodular eruption on the dorsum of the feet. There were no other abnormal physical findings.

The clinical diagnosis of meningococcal meningitis was confirmed when lumbar puncture revealed a turbid, yellow CSF at normal pressure. Staining revealed Gram-negative intracellular diplococci.
The CSF was then reported as showing 19200 leucocytes $\mathrm{mm}^{-3}(90 \%$ polymorphs), sugar of $0.05 \mathrm{mmol} / \mathrm{l}$, and protein of $2.8 \mathrm{~g} / 1$. CSF culture grew Neisseria meningitidis within 24 hours, subsequently reported as of Group B and sensitive to a range of antibiotics including penicillin, sulphonamides, chloramphenicol and ampicillin. Blood cultures taken at admission, before starting antibiotics, grew meningococcus after 48 hours incubation. In addition, $\mathrm{Hb}$ was $16.3 \mathrm{~g} / \mathrm{dl}$, white cell count $10900 \times 10^{9} / 1$, ESR $74 \mathrm{~mm}$ fall in one hour, platelets $150 \times 10^{9} / 1$, serum urea and electrolytes normal, and blood sugar $6.5 \mathrm{mmol} / 1$. Throat swab showed no growth.

Treatment initially was with benzylpenicillin 12 million units daily intravenously and sulphadimidine $1 \mathrm{~g}$ intravenously every 6 hours. Two days after admission the patient became pyrexial and chloramphenicol $1 \mathrm{~g}$ intramuscularly every 6 hours was added to the treatment.

A routine electrocardiogram on the day after admission showed sinus rhythm, with no gross conduction defect, but with significantly raised ST segments in most leads, viz. leads I, II, aVI, and V2-V6 (Fig.), indicative of pericarditis. However, the characteristic pericardial friction rub was not heard for a further 24 hours, when the heart sounds also became quieter, the apex beat impalpable, and cardiac dullness increased. A chest $x$-ray confirmed cardiomegaly, and cardiac screening was consistent with a moderate sized pericardial effusion. The patient complained only of a mild left-sided chest pain. A small volume of serous, blood-stained pericardial fluid was obtained by needle aspiration. No organisms were visible on 


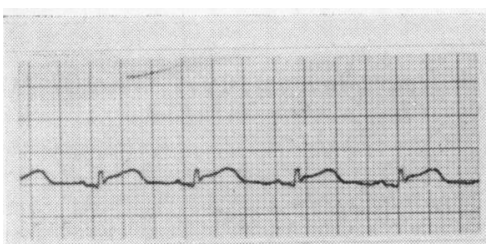

LEAD I

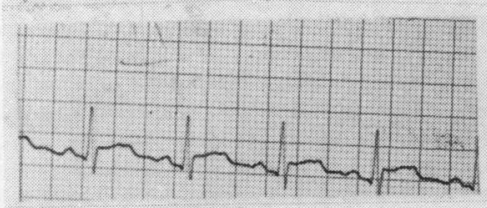

LEAD II

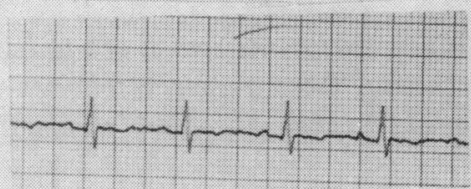

LEAD III

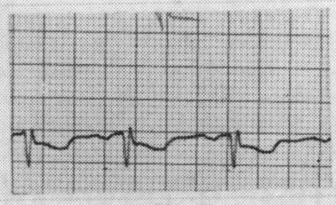

$\mathrm{aVr}$

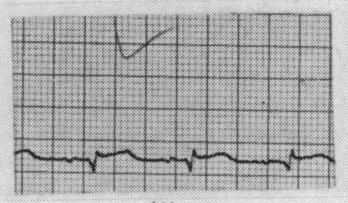

aVI

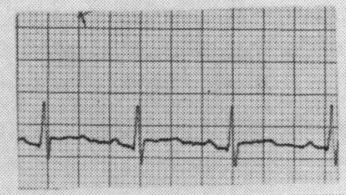

oVf

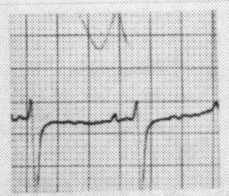

V1

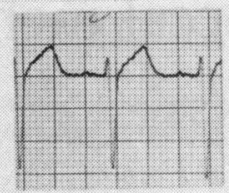

V2

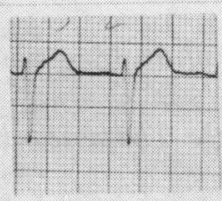

v3

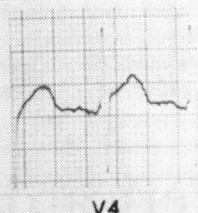

V4

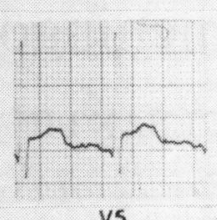

VS

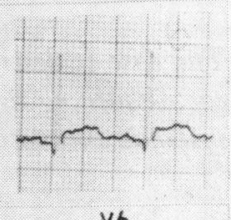

V6

FIG. Electrocardiogram on the day after admission showing raised $S T$ segments in $I, I I$, $a V 1$, and V2 to V6, indicative of pericarditis.

Gram staining, and culture was sterile. It had a total protein content of $45 \mathrm{~g} / \mathrm{l}$.

The patient gradually improved. The pericardial friction rub persisted for 21 days, while radiology and clinical examination confirmed reduction in the size of the effusion. The patient was discharged 30 days after admission in good health and with no evidence of cerebral damage. Follow-up visits failed to show any significant remaining cardiac disorder.

\section{Discussion}

Although generally regarded as being a rare complication in the late stages of the disease, the incidence of pericarditis in meningococcal meningitis varies from series to series. This is partly because of difficulties in diagnosis and somewhat differing diagnostic criteria. Dixon and Sanford (1971) found 17 cases of pericarditis in 334 cases of meningococcal meningitis $(5.1 \%)$, but in 5 of these cases the diagnosis was based strictly on electrocardiographic findings, with no reference to pain, friction rub, or radiological cardiomegaly. Herrick (1918) with 12 cases in 280 cases of meningitis $(4.3 \%)$, and Smithburn et al. (1936) with 9 cases in $140(6.4 \%)$, reported a similar incidence, but Morse, Ortesky, and Hudson (1971) found an incidence of 19 per cent ( 6 cases in 32 patients with meningitis). Their case material was unusual in that the patients were all young naval recruits. All positive cultures during the selected time were typed as Group C. The authors suggested that the organism involved had an altered antigenic capability to produce an inflammatory reaction on serosal membranes of an immunological rather than infective nature.

Certain of the complications of meningococcal disease, i.e. arthritis, cutaneous vasculitis, and episcleritis are thought by Whittle et al. (1973) and Greenwood, Whittle, and Bryceson (1973) to have an allergic basis. Pericarditis, however, is regarded as a direct septic complication of meningococcal meningitis with its associated meningococcaemia. Both Lukash (1963), and Lebowitz and Nespole (1967) attributed their cases of pericarditis complicating meningococcal meningitis to direct bacterial involvement rather than to any immunological mechanism. Their finding of a serous sterile effusion was thought to be the result of rapid transformation of a purulent effusion by systemic antibiotics crossing an inflamed pericardium. It is interesting to note that in both of these reports, clinical improvement followed the addition of systemic steroids to the antibiotic regimen. Experience of this single case does not allow the author to contribute to this debate. Formal pericardial drainage and the instillation of antibiotics into the pericardial space were not thought necessary in this instance, since cardiac compression was 
minimal and systemic antibiotics were considered to be crossing the pericardium in adequate concentrations.

The preceding malaise, arthritis, and skin rash of this case could be evidence of a preceding chronic meningococcaemia. This is a rare clinical entity and the classical clinical criteria are intermittent or sustained fevers associated with recurring maculopapular, nodular, or petechial eruptions, and migratory arthritis or arthralgia. The clinical features are probably caused by immune-complex deposition rather than by direct bacterial invasion (Liebel, Fangman, and Ostrovsky, 1974).

In reporting this case the author wishes to restate the need for routine performance of serial electrocardiograms on all cases of meningococcal meningitis, to help the early diagnosis of this rare but potentially fatal complication.

I am grateful to Dr. F. D. Rosenthal, consultant physician, for his permission to publish this case.

\section{References}

Dixon, L. M., and Sanford, H. S. (1971). Meningococcal pericarditis in the antibiotic era. Military Medicine, 136, 433.
Greenwood, B. M., Whittle, H. C., and Bryceson, A. D. M. (1973). Allergic complications of meningococcal disease. II. Immunological investigations. British Medical fournal, $2,73$.

Herrick, W. W. (1918). Meningococcic pericarditis with report of 12 cases. Medical Clinics of North America, 2, 411.

Lebowitz, W. B., and Nespole, A. S. (1967). Purulent pericarditis complicating meningococcal meningitis. American Fournal of Diseases of Children, 113, 385.

Leibel, R. L., Fangman, J. J., and Ostrovsky, M. C. (1974). Chronic meningococcemia in childhood. American fournal of Diseases of Children, 127, 94.

Lukash, W. M. (1963). Massive pericardial effusion due to meningococcic meningitis. fournal of the American Medical Association, 185, 598.

Morse, J. R., Ortesky, M. I., and Hudson, J. A. (1971). Pericarditis as a complication of meningococcal meningitis. Annals of Internal Medicine, 74, 212.

Smithburn, K. C., Kempt, G. F., Zergas, L. G., and Gilman, L. H. (1936). Meningococci meningitis. Fournal of the American Medical Association, 95, 776.

Whittle, H., Abdullahi, M. T., Fakunde, F. A., Greenwood, B. M., Bryceson, A. D. M., Parry, E. H. O., and Turk, J. L. (1973). Allergic complications of meningococcal disease. I. Clinical aspects. British Medical fournal, 2, 733.

Requests for reprints to Dr. Cleanth Jones, Leicester General Hospital, Gwendolen Road, Leicester LE5 4PW. 